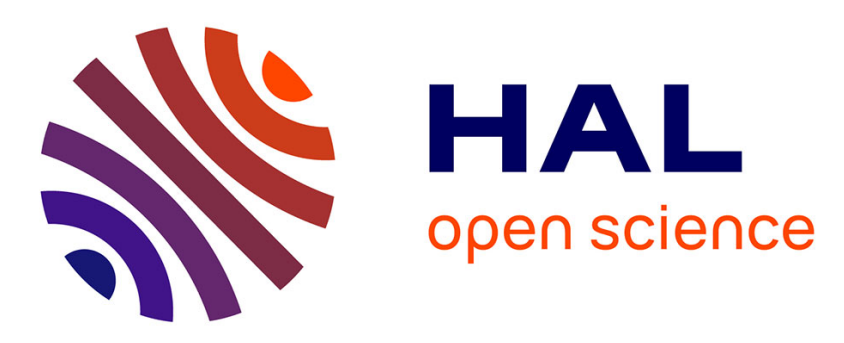

\title{
Signal subspace change detection in structured covariance matrices
}

R. Ben Abdallah, A. Breloy, A. Taylor, M. N. El Korso, David Lautru

\section{To cite this version:}

R. Ben Abdallah, A. Breloy, A. Taylor, M. N. El Korso, David Lautru. Signal subspace change detection in structured covariance matrices. 27th European Signal Processing Conference, Sep 2019, Coruna, Spain. hal-02333861

\section{HAL Id: hal-02333861 \\ https://hal.parisnanterre.fr/hal-02333861}

Submitted on 25 Oct 2019

HAL is a multi-disciplinary open access archive for the deposit and dissemination of scientific research documents, whether they are published or not. The documents may come from teaching and research institutions in France or abroad, or from public or private research centers.
L'archive ouverte pluridisciplinaire $\mathbf{H A L}$, est destinée au dépôt et à la diffusion de documents scientifiques de niveau recherche, publiés ou non, émanant des établissements d'enseignement et de recherche français ou étrangers, des laboratoires publics ou privés. 


\title{
Signal subspace change detection in structured covariance matrices
}

\author{
R. Ben Abdallah ${ }^{1}$, A. Breloy ${ }^{1}$, A. Taylor ${ }^{2}$, M. N. El Korso ${ }^{1}$ and D. Lautru ${ }^{1}$ \\ ${ }^{1}$ LEME (EA 4416), IUT de ville d'Avray, Université Paris Nanterre, France \\ ${ }^{2}$ DEMR, ONERA, Université Paris Saclay, F-91123 Palaiseau Cedex, France.
}

\begin{abstract}
Testing common properties between covariance matrices is a relevant approach in a plethora of applications. In this paper, we derive a new statistical test in the context of structured covariance matrices. Specifically, we consider low rank signal component plus white Gaussian noise structure. Our aim is to test the equality of the principal subspace, i.e., subspace spanned by the principal eigenvectors of a group of covariance matrices. A decision statistic is derived using the generalized likelihood ratio test. As the formulation of the proposed test implies a non-trivial optimization problem, we derive an appropriate majorizationminimization algorithm. Finally, numerical simulations illustrate the properties of the newly proposed detector compared to the state of the art.
\end{abstract}

Index Terms-Generalized likelihood ratio test, subspace testing, low rank structure, majorization-minimization algorithm.

\section{INTRODUCTION}

Testing common properties between covariance matrices is a classical problem in statistical signal processing [1]-[3]. For example, it has been applied in the context of radar and change detection in [4]-[8]. In this scope, the introduction of structure information on the covariance matrices can be relevant for the detection of specific underlying physical phenomenon [9], [10].

In this paper, we focus on CM modeled as a sum of low rank (LR) component (where the signal of interest lies in) plus scaled identity matrix. This structure, which is common for radar processing, has been successfully studied and applied to the context of radar detection [11]-[13]. Specifically, we are interested in designing a detection test that is only sensitive to a signal subspace variation. To this aim, we propose a decision statistic using the generalized likelihood ratio test (GLRT) based on the proposed binary hypothesis test. To compute the proposed test, we make use of the majorization minimization (MM) algorithm [13] for solving the optimization problems due to this GLRT. Finally, numerical simulations illustrate the performance of the proposed detection method.

The following notation is adopted along this paper: italic indicates a scalar quantity, lower case boldface indicates a vector quantity and upper case boldface a matrix. ${ }^{H}$ denotes the transpose conjugate operator or the simple conjugate operator for a scalar quantity. exptr\{.\} stands for exponential trace of a given matrix, $\mid$. $\mid$ denotes the determinant operator, $\mathcal{R}_{R}\{$.$\} is the range space spanned by the R$ principal eigenvectors of a given matrix defined formally in (7), $\mathcal{U}_{R}^{M}=\left\{\mathbf{U} \in \mathbb{C}^{M \times R} \mid \mathbf{U}^{H} \mathbf{U}=\mathbf{I}_{R}\right\}$ is the set of $M \times R$ semiunitary matrices, i.e., tall matrices whose columns form an orthonormal basis, $\mathcal{H}_{M}^{+}$is the set of $M \times M$ semi-definite Hermitian matrices and $\mathcal{G}_{R}$ is the set of rank $R$ orthogonal projectors.

\section{Problem Statement}

We consider $I+1$ independent sets of samples $\mathbf{z}_{k}^{i} \in \mathbb{C}^{M}$, $i \in \llbracket 0, I \rrbracket, k \in \llbracket 1, K \rrbracket$, with $K$ samples for each set $i$. The samples $\left\{\mathbf{z}_{k}^{i}\right\}$ are assumed i.i.d. w.r.t. the following model:

$$
\mathbf{z}_{k}^{i}=\mathbf{s}_{k}^{i}+\mathbf{n}_{k}^{i}
$$

- The signal $\mathbf{s}_{k}^{i} \sim \mathcal{C N}\left(0, \Sigma_{R}^{i}\right)$, in which the unknown scatter matrix, of rank $R \ll M$, is denoted as $\boldsymbol{\Sigma}_{R}^{i}=\mathbf{V}_{i} \mathbf{R}_{i} \mathbf{V}_{i}^{H}$ where $\mathbf{V}_{i} \in \mathcal{U}_{R}^{M}$ represents a signal subspace basis and $\mathbf{R}_{i} \in \mathbb{C}^{R \times R}$ is the signal $\mathrm{CM}$ in this space. The rank $R$ is assumed pre-established ${ }^{1}$. For example, such signal may model the ground response when considering correlation and/or power fluctuations (w.r.t. $i$ ).

- The white Gaussian noise $\mathbf{n}_{k}^{i} \sim \mathcal{C N}\left(0, \sigma^{2} \mathbf{I}\right)$ represents the contribution of thermal noise.

Eventually, the samples are drawn as $\mathbf{z}_{k}^{i} \sim \mathcal{C N}\left(0, \boldsymbol{\Sigma}_{i}\right)$, in which, $\boldsymbol{\Sigma}_{i}$ has the following expression:

$$
\boldsymbol{\Sigma}_{i}=\mathbf{V}_{i} \mathbf{R}_{i} \mathbf{V}_{i}^{H}+\sigma^{2} \mathbf{I} \triangleq \boldsymbol{\Sigma}_{R}^{i}+\sigma^{2} \mathbf{I}
$$

Consequently, the likelihood of the data set $\left\{\mathbf{z}_{k}^{i}\right\}$ reads as:

$$
\mathcal{L}\left(\left\{\mathbf{z}_{k}^{i}\right\} \mid \boldsymbol{\theta}\right)=\prod_{i=0}^{I} \frac{\operatorname{exptr}\left\{-\mathbf{S}_{i} \boldsymbol{\Sigma}_{i}^{-1}(\boldsymbol{\theta})\right\}}{\left|\boldsymbol{\Sigma}_{i}(\boldsymbol{\theta})\right|^{K}}
$$

where $\mathbf{S}_{i}=\sum_{k=1}^{K} \mathbf{z}_{k}^{i} \mathbf{z}_{k}^{i^{H}}$ and $\boldsymbol{\theta}$ is an appropriate CM parameterization of the set $\left\{\boldsymbol{\Sigma}_{i}\right\}$. In this paper, we study the problem of testing whether the set under test, namely $i=0$, shares common properties with the secondary sets. Specifically, for the general model (1), we propose a novel detector based on testing the equality of range spaces $\mathcal{R}_{R}\left\{\boldsymbol{\Sigma}_{i}\right\}$.

\section{RELATED WORKS}

In this Section, we present two standards GLRT used for testing the similarity of CMs. The first test is for the equality of $\mathrm{CMs}$, while the second one is derived for the proportionality testing between CMs.

\footnotetext{
${ }^{1}$ Indeed, the proposed results can be applied using plug-in rank estimates or by integrating physical prior knowledge on this parameter [14]. About rank estimation, the reader is refered to the overview [15] and recent methods using shrinkage [16] or random matrix theory [17].
} 


\section{A. Equality testing}

The general problem of testing $\mathrm{CM}$ equality [1] in the complex-valued Gaussian case is analyzed in e.g. [4]. The hypothesis test, for $\mathbf{z}_{k}^{i} \sim \mathcal{C N}\left(0, \boldsymbol{\Sigma}_{i}\right)$, reads as:

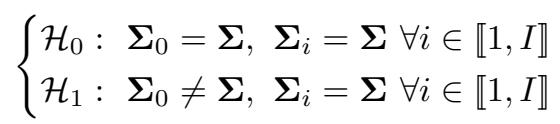

The corresponding GLRT for the above test is given in [4]. The LR counterpart of this test (for structured CMs as in (2)) has been proposed in [10].

\section{B. Proportionality testing}

The general problem of testing CM proportionality [2] in the complex-valued Gaussian case is analyzed in e.g. [9]. For $\mathbf{z}_{k}^{i} \sim \mathcal{C N}\left(0, \boldsymbol{\Sigma}_{i}\right)$, the hypothesis test is:

$$
\left\{\begin{array}{l}
\mathcal{H}_{0}: \boldsymbol{\Sigma}_{0}=\beta_{0} \boldsymbol{\Sigma}, \boldsymbol{\Sigma}_{i}=\beta_{i} \boldsymbol{\Sigma} \forall i \in \llbracket 1, I \rrbracket \\
\mathcal{H}_{1}: \boldsymbol{\Sigma}_{0} \neq \beta_{0} \boldsymbol{\Sigma}, \boldsymbol{\Sigma}_{i}=\beta_{i} \boldsymbol{\Sigma} \forall i \in \llbracket 1, I \rrbracket
\end{array}\right.
$$

The corresponding GLRT for this hypothesis test is given in [9]. Again, a LR counterpart of this test has been proposed in [10].

\section{Contribution of this paper}

The aformentioned detectors are based on CM equality or proportionality testing. Conversely, we focus here on the signal subspace equality testing, i.e., we aim to build a test that accounts only for a change in the signal subspace for CMs as in (2). Specifically, we will test the following hypothesis:

$$
\left\{\begin{array}{l}
\mathcal{H}_{0}: \mid \mathcal{R}_{R}\left\{\boldsymbol{\Sigma}_{i}\right\}=\mathcal{R}_{R}\{\boldsymbol{\Sigma}\}, \forall i \in \llbracket 0, I \rrbracket \\
\mathcal{H}_{1}: \mid \begin{array}{l}
\mathcal{R}_{R}\left\{\boldsymbol{\Sigma}_{i}\right\}=\mathcal{R}_{R}\{\boldsymbol{\Sigma}\}, \forall i \in \llbracket 1, I \rrbracket \\
\mathcal{R}_{R}\left\{\boldsymbol{\Sigma}_{0}\right\} \neq \mathcal{R}_{R}\{\boldsymbol{\Sigma}\}
\end{array}
\end{array}\right.
$$

where $\mathcal{R}_{R}\{$.$\} is the range space spanned by the R$ principal eigenvectors of a given matrix, defined by the operator

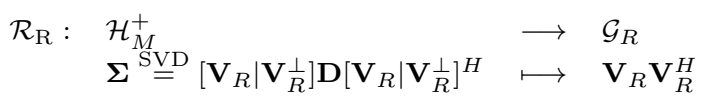

Remark: This proposed test can assess for a specific underlying physical mechanism. For example, in a radar context the source power can fluctuate (change in the signal CMs $\mathbf{R}_{i}$ in (2)) while spanning the same signal subspace. This leads to both:

$$
\boldsymbol{\Sigma}_{0} \neq \boldsymbol{\Sigma}_{i} \text { and } \boldsymbol{\Sigma}_{0} \not \subset \boldsymbol{\Sigma}_{i}, \forall i
$$

and

$$
\mathcal{R}_{R}\left\{\boldsymbol{\Sigma}_{0}\right\}=\mathcal{R}_{R}\left\{\boldsymbol{\Sigma}_{i}\right\}, \forall i
$$

Notice that (8) is considered as $\mathcal{H}_{1}$ for the standard tests (4) and (5) while the relation (9) gives $\mathcal{H}_{0}$ for the test (6). Thus, the proposed detector is insensitive to the sources correlations/power fluctuations, which can lead to lower false alarm rates in specific detection applications [9].

\section{Proposed DETECTOR \& ALGORITHM DERIVATION}

We test whether the sample sets share the same principal subspace. From (2) and (6), the hypothesis test can be reformulated as:

$$
\left\{\begin{array}{l}
\mathcal{H}_{0}: \mid \boldsymbol{\Sigma}_{i}=\mathbf{V}_{\mathcal{H}_{0}} \mathbf{R}_{\mathcal{H}_{0}}^{i}\left(\mathbf{V}_{\mathcal{H}_{0}}\right)^{H}+\sigma^{2} \mathbf{I}, \quad \forall i \in \llbracket 0, I \rrbracket \\
\mathcal{H}_{1}: \mid \begin{array}{l}
\boldsymbol{\Sigma}_{i}=\mathbf{V}_{\mathcal{H}_{1}}^{\star} \mathbf{R}_{\mathcal{H}_{1}}^{i}\left(\mathbf{V}_{\mathcal{H}_{1}}^{\star}\right)^{H}+\sigma^{2} \mathbf{I}, \quad \forall i \in \llbracket 1, I \rrbracket \\
\boldsymbol{\Sigma}_{0}=\mathbf{V}_{\mathcal{H}_{1}}^{0} \mathbf{R}_{\mathcal{H}_{1}}^{0}\left(\mathbf{V}_{\mathcal{H}_{1}}^{0}\right)^{H}+\sigma^{2} \mathbf{I}
\end{array}
\end{array}\right.
$$

where $\mathbf{V}_{\mathcal{H}_{0}}\left(\mathbf{V}_{\mathcal{H}_{0}}\right)^{H}, \mathbf{V}_{\mathcal{H}_{1}}^{\star}\left(\mathbf{V}_{\mathcal{H}_{1}}^{\star}\right)^{H}$ and $\mathbf{V}_{\mathcal{H}_{1}}^{0}\left(\mathbf{V}_{\mathcal{H}_{1}}^{0}\right)^{H}$ correspond to the range spaces of respectively the secondary sets under $\mathcal{H}_{0}$, under $\mathcal{H}_{1}$ and of the tested set under $\mathcal{H}_{1}$. The quantities $\mathbf{R}_{\mathcal{H}_{0}}^{i}, \mathbf{R}_{\mathcal{H}_{1}}^{i}$ and $\mathbf{R}_{\mathcal{H}_{1}}^{0}$ denote the signal CM in LR subspace of respectively the secondary sets under $\mathcal{H}_{0}$, under $\mathcal{H}_{1}$ and the tested set under $\mathcal{H}_{1}$. The GLRT for principal subspace equality is given as:

$$
\frac{\max _{\boldsymbol{\theta}_{\mathcal{H}_{1}}^{\text {sub }}} \mathcal{L}\left(\left\{\mathbf{z}_{k}^{i}\right\} \mid \mathcal{H}_{1}, \boldsymbol{\theta}_{\mathcal{H}_{1}}^{\text {sub }}\right)}{\max _{\boldsymbol{\theta}_{\mathcal{H}_{0}}^{\text {sub }}} \mathcal{L}\left(\left\{\mathbf{z}_{k}^{i}\right\} \mid \mathcal{H}_{0}, \boldsymbol{\theta}_{\mathcal{H}_{0}}^{\text {sub }}\right)} \underbrace{\stackrel{\mathcal{H}_{1}}{\gtrless}}_{\mathcal{H}_{0}} \delta_{\text {glr }}^{\text {sub }}
$$

with sets of parameters

$$
\begin{aligned}
& \boldsymbol{\theta}_{\mathcal{H}_{0}}^{\text {sub }}=\left\{\left\{\mathbf{R}_{\mathcal{H}_{0}}^{i}\right\}_{i \in \llbracket 0, I \rrbracket}, \mathbf{V}_{\mathcal{H}_{0}}\right\} \\
& \boldsymbol{\theta}_{\mathcal{H}_{1}}^{\text {sub }}=\left\{\left\{\mathbf{R}_{\mathcal{H}_{1}}^{i}\right\}_{i \in \llbracket 0, I \rrbracket}, \mathbf{V}_{\mathcal{H}_{1}}^{\star}, \mathbf{V}_{\mathcal{H}_{1}}^{0}\right\}
\end{aligned}
$$

and where functions $\mathcal{L}\left(\left\{\mathbf{z}_{k}^{i}\right\} \mid \mathcal{H}_{1}, \boldsymbol{\theta}_{\mathcal{H}_{1}}^{\text {sub }}\right), \mathcal{L}\left(\left\{\mathbf{z}_{k}^{i}\right\} \mid \mathcal{H}_{0}, \boldsymbol{\theta}_{\mathcal{H}_{0}}^{\text {sub }}\right)$ denote the likelihood of the dataset $\left\{\mathbf{z}_{k}^{i}\right\}$ under respectively $\mathcal{H}_{1}$ and $\mathcal{H}_{0}$. Then, the GLRT for the proposed test reads as:

$$
\frac{\mathcal{L}\left(\left\{\mathbf{z}_{k}^{i}\right\} \mid \mathcal{H}_{1}, \widehat{\boldsymbol{\theta}}_{\mathcal{H}_{1}}^{\text {sub }}\right)}{\mathcal{L}\left(\left\{\mathbf{z}_{k}^{i}\right\} \mid \mathcal{H}_{0}, \widehat{\boldsymbol{\theta}}_{\mathcal{H}_{0}}^{\text {sub }}\right)} \underset{\mathcal{H}_{0}}{\stackrel{\mathcal{H}_{1}}{\gtrless}} \delta_{\mathrm{glr}}^{\text {sub }}
$$

where $\widehat{\boldsymbol{\theta}}_{\mathcal{H}_{1}}^{\text {sub }}$ and $\widehat{\boldsymbol{\theta}}_{\mathcal{H}_{0}}^{\text {sub }}$ are the maximum likelihood estimators (MLE) of respectively $\boldsymbol{\theta}_{\mathcal{H}_{1}}^{\text {sub }}$ and $\boldsymbol{\theta}_{\mathcal{H}_{0}}^{\text {sub }}$. In order to evaluate this GLRT, we design in the following block-coordinate descent algorithms to compute the MLE of $\boldsymbol{\theta}_{\mathcal{H}_{0}}^{\text {sub }}$ and $\boldsymbol{\theta}_{\mathcal{H}_{1}}^{\text {sub }}$. Specifically, we make use of the block MM algorithm [18] for this problem. This algorithm performs a block coordinate update of the parameters by minimizing the surrogate (majorizing) function of the objective. The interest of the majorization lies in the possibility to obtain closed form updates and to ensure a monotonic decrement of the objective value at each step. There are general no results on the convergence towards the global minimum, but good performance is observed in practice. Furthermore, the convergence of the objective function is guaranteed under certain mild conditions [19].

\section{A. MLE of $\boldsymbol{\theta}_{\mathcal{H}_{0}}^{\text {sub }}$ under $\mathcal{H}_{0}$}

Under $\mathcal{H}_{0}$, the likelihood optimization reduces to:

$$
\begin{array}{ll}
\max _{\boldsymbol{\theta}_{\mathcal{H}_{0}}^{\text {sub }}} & \mathcal{L}\left(\left\{\mathbf{z}_{k}^{i}\right\} \mid \mathcal{H}_{0}, \boldsymbol{\theta}_{\mathcal{H}_{0}}^{\text {sub }}\right) \\
\text { s. t. } & \mathbf{R}_{\mathcal{H}}^{i} \succcurlyeq \mathbf{0}, \forall i \in \llbracket 0, I \rrbracket \\
& \mathbf{V}_{\mathcal{H}_{0}}^{H} \mathbf{V}_{\mathcal{H}_{0}}=\mathbf{I}
\end{array}
$$


This problem is equivalent to minimizing the negative loglikelihood as:

$$
\begin{array}{ll}
\min _{\boldsymbol{\theta}_{\mathcal{H}_{0}}^{\text {sub }}} & \sum_{i=0}^{I}\left[K \ln \left(\left|\boldsymbol{\Sigma}_{i}\right|\right)+\operatorname{Tr}\left\{\mathbf{S}_{i} \boldsymbol{\Sigma}_{i}^{-1}\right\}\right] \\
\text { s. t. } & \boldsymbol{\Sigma}_{i}=\mathbf{V}_{\mathcal{H}_{0}} \mathbf{R}_{\mathcal{H}_{0}}^{i}\left(\mathbf{V}_{\mathcal{H}_{0}}\right)^{H}+\sigma^{2} \mathbf{I}, \forall i \in \llbracket 0, I \rrbracket \\
& \mathbf{R}_{\mathcal{H}_{0}}^{i} \succcurlyeq \mathbf{0} \\
& \mathbf{V}_{\mathcal{H}_{0}}^{H} \mathbf{V}_{\mathcal{H}_{0}}=\mathbf{I}
\end{array}
$$

To solve this problem, we derive an iterative alterning algorithm that sequentially updates the variables $\left\{\mathbf{R}_{\mathcal{H}_{0}}^{i}\right\}$ and $\mathbf{V}_{\mathcal{H}_{0}}$. The main steps of the estimation process are summed up in the box Algorithm 1, and are briefly explained in the following.

1) Update $\left\{\mathbf{R}_{\mathcal{H}_{0}}^{i}\right\}$ : Considering $\left\{\mathbf{R}_{\mathcal{H}_{0}}^{i}\right\}$ while fixing the remain variables, the problem in (15) is separable for each $\mathbf{R}_{\mathcal{H}_{0}}^{i}$. The objective of (15) w.r.t. the variable $\mathbf{R}_{\mathcal{H}_{0}}^{i}$ can be expressed as:

$$
\mathcal{L}_{r}\left(\mathbf{R}_{\mathcal{H}_{0}}^{i}\right)=K \ln \left|\left(\mathbf{R}_{\mathcal{H}_{0}}^{i}+\sigma^{2} \mathbf{I}\right)\right| \operatorname{Tr}\left\{\tilde{\mathbf{S}}_{i}\left(\mathbf{R}_{\mathcal{H}_{0}}^{i}+\sigma^{2} \mathbf{I}\right)^{-1}\right\}
$$

with $\tilde{\mathbf{S}}_{i}=\mathbf{V}_{\mathcal{H}_{0}}^{H} \mathbf{S}_{i} \mathbf{V}_{\mathcal{H}_{0}}$. The minimizer of this objective w.r.t. $\mathbf{R}_{\mathcal{H}_{0}}^{i}$ corresponds therefore to the MLE of a structured CM for dimension reduced Gaussian variables [20]. Denoting the eigenvalue decomposition (EVD) of the dimension reduced SCM as:

$$
\tilde{\mathbf{S}}_{i} / K \stackrel{\mathrm{EVD}}{=} \mathbf{Q}_{R} \mathbf{D}_{R} \mathbf{Q}_{R}^{H}
$$

Thus, the update is given as:

$$
\left\{\begin{array}{l}
\mathbf{R}_{\mathcal{H}_{0}}^{i(t+1)}=\mathbf{Q}_{R} \tilde{\mathbf{D}}_{R} \mathbf{Q}_{R}^{H} \\
{\left[\tilde{\mathbf{D}}_{R}\right]_{r, r}=\max \left(\left[\mathbf{D}_{R}\right]_{r, r}-\sigma^{2}, 0\right), \forall r \in \llbracket 1, R \rrbracket}
\end{array}\right.
$$

2) Update $\mathbf{V}_{\mathcal{H}_{0}}$ : First, remark that $\boldsymbol{\Sigma}_{i}^{-1}$ can be expressed thanks to the matrix inversion lemma as:

$$
\begin{aligned}
\boldsymbol{\Sigma}_{i}^{-1} & =\left(\mathbf{V}_{\mathcal{H}_{0}} \mathbf{R}_{\mathcal{H}_{0}}^{i}\left(\mathbf{V}_{\mathcal{H}_{0}}\right)^{H}+\sigma^{2} \mathbf{I}\right)^{-1} \\
& =\sigma^{-2} \mathbf{I}-\mathbf{V}_{\mathcal{H}_{0}} \underbrace{\sigma^{-4}\left(\left(\mathbf{R}_{\mathcal{H}_{0}}^{i}\right)^{-1}+\sigma^{-2} \mathbf{I}\right)^{-1}}_{\mathbf{W}_{i}} \mathbf{V}_{\mathcal{H}_{0}}^{H}
\end{aligned}
$$

After some calculus, the objective in (15) w.r.t. $\mathbf{V}_{\mathcal{H}_{0}}$ for other fixed variables can be expressed:

$$
f\left(\mathbf{V}_{\mathcal{H}_{0}}\right)=\sum_{i=0}^{I} \operatorname{Tr}\left\{\left(\mathbf{V}_{\mathcal{H}_{0}}\right)^{H} \mathbf{S}_{i} \mathbf{V}_{\mathcal{H}_{0}} \mathbf{W}_{i}\right\}
$$

An update of $\mathbf{V}_{\mathcal{H}_{0}}$ can be obtained in closed form by following the MM approach [18]. Applying the proposition 3 of [13], the objective can be majorized with equality at point $\mathbf{V}_{\mathcal{H}_{0}}^{(t)}$ as:

$$
f\left(\mathbf{V}_{\mathcal{H}_{0}}\right) \leq g\left(\mathbf{V}_{\mathcal{H}_{0}} \mid \mathbf{V}_{\mathcal{H}_{0}}^{(t)}\right)
$$

with

$$
g\left(\mathbf{V}_{\mathcal{H}_{0}} \mid \mathbf{V}_{\mathcal{H}_{0}}^{(t)}\right)=\sum_{i=1}^{I} 2 \operatorname{Re}\left[\operatorname{Tr}\left\{\left(\mathbf{V}_{\mathcal{H}_{0}}\right)^{H} \mathbf{S}_{i}\left(\mathbf{V}_{\mathcal{H}_{0}}\right)^{(t)} \mathbf{W}_{i}\right\}\right]
$$

Then, $\mathbf{V}_{\mathcal{H}_{0}}^{(t+1)}$ is obtained as the minimizer of the following problem:

$$
\begin{array}{ll}
\min _{\mathbf{V}_{\mathcal{H}_{0}}} & g\left(\mathbf{V}_{\mathcal{H}_{0}} \mid \mathbf{V}_{\mathcal{H}_{0}}^{(t)}\right) \\
\text { s. t. } & \mathbf{V}_{\mathcal{H}_{0}}^{H} \mathbf{V}_{\mathcal{H}_{0}}=\mathbf{I}
\end{array}
$$

Solving this optimization problem under the orthonormality constraint leads to an update of the form:

$$
\mathbf{V}_{\mathcal{H}_{0}}^{(t+1)}=\mathbf{U}_{\text {left }} \mathbf{U}_{\text {right }}^{H}
$$

with $\mathbf{U}_{\text {left }}$ and $\mathbf{U}_{\text {right }}^{H}$ are the left and right eigenvectors of the thin singular value decomposition (TSVD), that is:

$$
\sum_{i=0}^{I}\left(\mathbf{S}_{i} \mathbf{V}_{\mathcal{H}_{0}}^{(t)} \mathbf{W}_{i}\right) \stackrel{\mathrm{TSVD}}{=} \mathbf{U}_{\text {left }} \mathbf{D} \mathbf{U}_{\text {right }}^{H}
$$

\section{B. MLE of $\boldsymbol{\theta}_{\mathcal{H}_{1}}^{\text {sub }}$ under $\mathcal{H}_{1}$}

Under $\mathcal{H}_{1}$, the optimization problem reads:

$$
\begin{array}{cl}
\max _{\boldsymbol{\theta}_{\mathcal{H}_{1}}^{\text {sub }}} & \mathcal{L}\left(\left\{\mathbf{z}_{k}^{i}\right\} \mid \mathcal{H}_{1}, \boldsymbol{\theta}_{\mathcal{H}_{1}}^{\text {sub }}\right) \\
\text { s. t. } & \mathbf{R}_{\mathcal{H}_{0}}^{i} \succcurlyeq \mathbf{0} \\
& \left(\mathbf{V}_{\mathcal{H}_{1}}^{0}\right)^{H} \mathbf{V}_{\mathcal{H}_{1}}^{0}=\mathbf{I} \\
& \left(\mathbf{V}_{\mathcal{H}_{1}}^{\star}\right)^{H} \mathbf{V}_{\mathcal{H}_{1}}^{\star}=\mathbf{I}
\end{array}
$$

which is seperable in $\left\{\left\{\mathbf{R}_{\mathcal{H}_{1}}^{i}\right\}_{i \in \llbracket 1, I \rrbracket}, \quad \mathbf{V}_{\mathcal{H}_{1}}^{\star}\right\} \quad$ and $\left\{\mathbf{R}_{\mathcal{H}_{1}}^{0}, \mathbf{V}_{\mathcal{H}_{1}}^{0}\right\}$, for which we derive appropriate solutions in the following. The corresponding algorithm is summed up in the box Algorithm 2 and a brief explanation of the procedure updates are given below.

1) MLE of $\left\{\left\{\mathbf{R}_{\mathcal{H}_{1}}^{i}\right\}_{i \in \llbracket 1, I \rrbracket}, \mathbf{V}_{\mathcal{H}_{1}}^{\star}\right\}$ under $\mathcal{H}_{1}$ : This problem is identical to (15) except that the set $i=0$ is excluded. Hence, we can directly use Algorithm 1 to obtain the solutions $\left\{\hat{\mathbf{R}}_{\mathcal{H}_{1}}^{i}\right\}_{i \in \llbracket 1, I \rrbracket}$ and $\hat{\mathbf{V}}_{\mathcal{H}_{1}}^{\star}$.

2) MLE of $\left\{\mathbf{R}_{\mathcal{H}_{1}}^{0}, \mathbf{V}_{\mathcal{H}_{1}}^{0}\right\}$ under $\mathcal{H}_{1}$ : This problem reduces to:

$$
\begin{array}{cl}
\min _{\mathbf{R}_{\mathcal{H}_{1}}^{0}, \mathbf{V}_{\mathcal{H}_{1}}^{0}} & K \ln \left(\left|\boldsymbol{\Sigma}_{i}\right|\right)+\operatorname{Tr}\left\{\mathbf{S}_{i} \boldsymbol{\Sigma}_{i}^{-1}\right\} \\
\text { s. t. } & \boldsymbol{\Sigma}_{0}=\mathbf{V}_{\mathcal{H}_{1}}^{0} \mathbf{R}_{\mathcal{H}_{1}}^{0}\left(\mathbf{V}_{\mathcal{H}_{1}}^{0}\right)^{H}+\sigma^{2} \mathbf{I} \\
& \mathbf{R}_{\mathcal{H}_{1}}^{0} \succcurlyeq \mathbf{0} \\
& \left(\mathbf{V}_{\mathcal{H}_{1}}^{0}\right)^{H} \mathbf{V}_{\mathcal{H}_{1}}^{0}=\mathbf{I}
\end{array}
$$

Then, the solution corresponds to the MLE of the LR structured CM in the context of Gaussian data [20]. Let us denote the EVD of the SCM as follows:

$$
\mathbf{S}_{0} / K \stackrel{\mathrm{EVD}}{=}\left[\mathbf{U}_{R} \mathbf{U}_{R}^{\perp}\right]\left[\begin{array}{cc}
\mathbf{D}_{R} & \mathbf{0} \\
\mathbf{0} & \mathbf{D}_{M-R}
\end{array}\right]\left[\begin{array}{ll}
\mathbf{U}_{R} & \mathbf{U}_{R}^{\perp}
\end{array}\right]^{H}
$$

The solution reads:

$$
\left\{\begin{array}{l}
{\left[\hat{\mathbf{R}}_{\mathcal{H}_{1}}^{0}\right]_{r, r}=\max \left(\left[\mathbf{D}_{R}\right]_{r, r}-\sigma^{2}, 0\right), \forall r \in \llbracket 1, R \rrbracket} \\
\hat{\mathbf{V}}_{\mathcal{H}_{1}}^{0}=\mathbf{U}_{R}
\end{array}\right.
$$

\section{Numerical Simulations}

\section{A. Simulation setup}

This section presents numerical simulations to assess the performance of the proposed GLRT detector for the subspace equality, denoted by $t_{\text {sub }}$, compared to the following detectors:

- $t_{\mathrm{E}}$ stands for the GLRT for the equality testing [4].

- $t_{\mathrm{E}}^{\mathrm{LR}}$ denotes the GLRT for the LR structured CM equality testing [10].

- $t_{\mathrm{P}}$ denotes the GLRT for the proportionality testing [9]. 

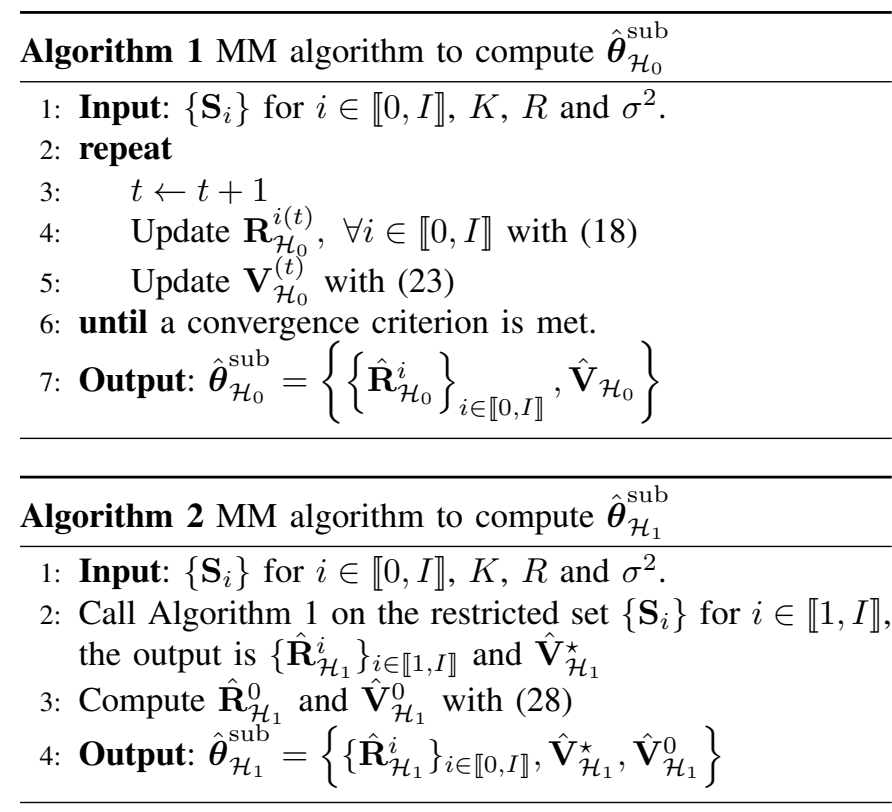

- $t_{\mathrm{P}}^{\mathrm{LR}}$ is the GLRT for testing the proportionality of LR signal CM component [10].

To this end, we consider, $M=20, R=5, K=25$, $I=3$, the samples $\mathbf{z}_{k}^{i}$ are drawn from i.i.d. complex normal distribution, i.e., $\mathbf{z}_{k}^{i} \sim \mathcal{C N}\left(0, \boldsymbol{\Sigma}_{i}\right)$ and $\boldsymbol{\Sigma}_{i}=\tau_{i} \mathbf{V}_{i} \boldsymbol{\Lambda} \mathbf{V}_{i}^{H}+\sigma^{2} \mathbf{I}$ where $\mathbf{V}_{i} \in \mathcal{U}_{R}^{M}, \tau_{i} \in \mathbb{R}^{+}$and $\boldsymbol{\Lambda}$ is a diagonal matrix. The eigenvalues $[\boldsymbol{\Lambda}]_{r, r}=\alpha(R+1-r)$, the signal to noise ratio $\mathrm{SNR}=\operatorname{Tr}\{\boldsymbol{\Lambda}\} / R \sigma^{2}$ with $\sigma^{2}=1$. As comparatif creteria, we consider the the receiver operating characteristic (ROC) curve which displays the probability of detection (PD) versus the probability of false alarm (PFA) for the following scenarios:

- Scenario 1 : Under $\mathcal{H}_{0}$ and $\mathcal{H}_{1}, \tau_{i}=1$ and $\mathbf{V}_{i}=\mathbf{V}, \forall i \in$ $\llbracket 1, I \rrbracket$ where $\mathbf{V} \in \mathcal{U}_{R}^{M}$ is built from the $R$ first elements of the canonical basis. The CM of the tested set, under $\mathcal{H}_{1}$, reads as $\boldsymbol{\Sigma}_{0}=\mathbf{V}_{0} \boldsymbol{\Lambda} \mathbf{V}_{0}^{H}+\sigma^{2} \mathbf{I}$ where $\mathbf{V}_{0} \in \mathcal{U}_{R}^{M}$ is generated by changing the first 2 eigenvectors of $\mathbf{V}$, i.e., $\mathbf{V}_{0} \neq \mathbf{V}$ so that $\mathcal{R}_{R}\left\{\boldsymbol{\Sigma}_{0}\right\} \neq \mathcal{R}_{R}\left\{\boldsymbol{\Sigma}_{i}\right\}, \forall i$. This scenario corresponds to a strict change in the $\mathrm{CM}$ where all the secondary sets are homogeneous.

- Scenario 2: Under $\mathcal{H}_{0}$ and $\mathcal{H}_{1}, \tau_{i} \sim \Gamma(\nu, 1 / \nu)$ (with $\nu=$ 1) and $\mathbf{V}_{i}=\mathbf{V}, \forall i \in \llbracket 1, I \rrbracket$ where $\mathbf{V} \in \mathcal{U}_{R}^{M}$ is built from the $R$ first elements of the canonical basis. Under $\mathcal{H}_{1}$, the anomaly in the CM of the tested set $\boldsymbol{\Sigma}_{0}=\mathbf{V}_{0} \boldsymbol{\Lambda} \mathbf{V}_{0}^{H}+\sigma^{2} \mathbf{I}$ is generated again by changing its principal subspace, i.e., $\mathcal{R}_{R}\left\{\boldsymbol{\Sigma}_{0}\right\} \neq \mathcal{R}_{R}\left\{\boldsymbol{\Sigma}_{i}\right\}, \forall i$ as in Scenario 1. This scenario accounts for power fluctuations of the signal w.r.t. the batches $i$. Hence, the signal $\mathrm{CM}$ are proportional under $\mathcal{H}_{0}$, but the total CMs are not identical.

- Scenario 3 : Under $\mathcal{H}_{0}$ and $\mathcal{H}_{1}, \tau_{i} \sim \Gamma(\nu, 1 / \nu)$ (with $\nu=1)$ and $\mathbf{V}_{i}=\mathbf{V Q}_{i}, \forall i \in \llbracket 1, I \rrbracket$ where $\mathbf{V} \in \mathcal{U}_{R}^{M}$ is built from the $R$ first elements of the canonical basis and $\left\{\mathbf{Q}_{i}\right\}$ is a set of $R \times R$ rotation matrices. We note that, under $\mathcal{H}_{0}$, we have (8) while the relation (7) is also satisfied. The CM of the tested set is $\boldsymbol{\Sigma}_{0}=\mathbf{V}_{0} \boldsymbol{\Lambda} \mathbf{V}_{0}^{H}+\sigma^{2} \mathbf{I}$, under $\mathcal{H}_{1}$, which corresponds to a change in its principal subspace where $\mathcal{R}_{R}\left\{\boldsymbol{\Sigma}_{0}\right\} \neq \mathcal{R}_{R}\left\{\boldsymbol{\Sigma}_{i}\right\}, \forall i$ as in the other scenarios. Scenario 3 aims to test a specific physical phenomenon as discussed in Section III-C.

\section{B. Results}

Figure 1 presents the ROC of the different detectors under various scenarios and SNRs:

- The first column (left) displays the ROC curves of the detectors for Scenario 1 for respectively $\mathrm{SNR}=0 \mathrm{~dB}$ (top) and $\mathrm{SNR}=5 \mathrm{~dB}$ (bottom). Under this setting, $t_{\mathrm{P}}$ and $t_{\mathrm{E}}$ appear to have identical performance. The proposed detector $t_{\mathrm{sub}}$ outperforms $t_{\mathrm{P}}$ and $t_{\mathrm{E}}$ since it exploits the LR structure information. However, the detectors $t_{\mathrm{P}}^{\mathrm{LR}}$ and $t_{\mathrm{E}}^{\mathrm{LR}}$ outperform all the others. This result was to be expected since these two detectors are specifically suited to this scenario $\left(t_{\mathrm{E}}^{\mathrm{LR}}\right.$ corresponds to the GLRT for this exact setting). We note that at $\mathrm{SNR}=5 \mathrm{~dB}$, the change detection problem is not especially challenging in this setting, thus all detectors show high performance, i.e., $\mathrm{PD} \approx 1$ for $\mathrm{PFA} \approx 10^{-4}$.

- The second column (central) displays the ROC curves of detectors for Scenario 2 for respectively $\mathrm{SNR}=0 \mathrm{~dB}$ (top) and $\mathrm{SNR}=5 \mathrm{~dB}$ (bottom). In this setting LR proportionality detector $t_{\mathrm{P}}^{\mathrm{LR}}$ corresponds to the most appropriate GLRT, thus it outperforms the other detectors. An explanation is that even under $\mathcal{H}_{0}$, the total CMs of the different batches verify (8) (non-equal and non-proportional). Thus, $t_{\mathrm{E}}, t_{\mathrm{P}}$, and $t_{\mathrm{E}}^{\mathrm{LR}}$ require a high detection threshold to ensure a low PFA. It is worth mentioning that we are mostly interested in the performance of the detectors for low PFA (below 0.1). In this range, $t_{\mathrm{sub}}$ also offers interesting performance since it is, as $t_{\mathrm{P}}^{\mathrm{LR}}$, designed to be insensitive to power fluctuations of the LR signal CM.

- The third (right) column displays the ROC curves of detectors for Scenario 3 for respectively $\mathrm{SNR}=0 \mathrm{~dB}$ (top) and $\mathrm{SNR}=5 \mathrm{~dB}$ (bottom). In this setting, the proposed detector $t_{\text {sub }}$ outperforms $t_{\mathrm{E}}, t_{\mathrm{E}}^{\mathrm{LR}}, t_{\mathrm{P}}$ and $t_{\mathrm{P}}^{\mathrm{LR}}$, since equality, LR equality, proportionality and LR proportionality detectors do not directly infer on the equality of signal subspace in the context of LR structure CMs. For the considered scenario, the correlation between the signal components change w.r.t. $i$ even under $\mathcal{H}_{0}$. However, (7) is satisfied so $t_{\text {sub }}$ is not sensitive to this type of heterogeneity. Therefore, this detector allows to reduce the PFA as discussed in Section III-C.

\section{CONCLUSION}

This paper introduced a new detector that is only sensitive to the variation of the signal subspace in the context of LR structure CM. This new detection method is relevant for reducing the false alarm rate when the signal has a varying CM (e.g. power fluctuations) but lies in a stationary subspace. 

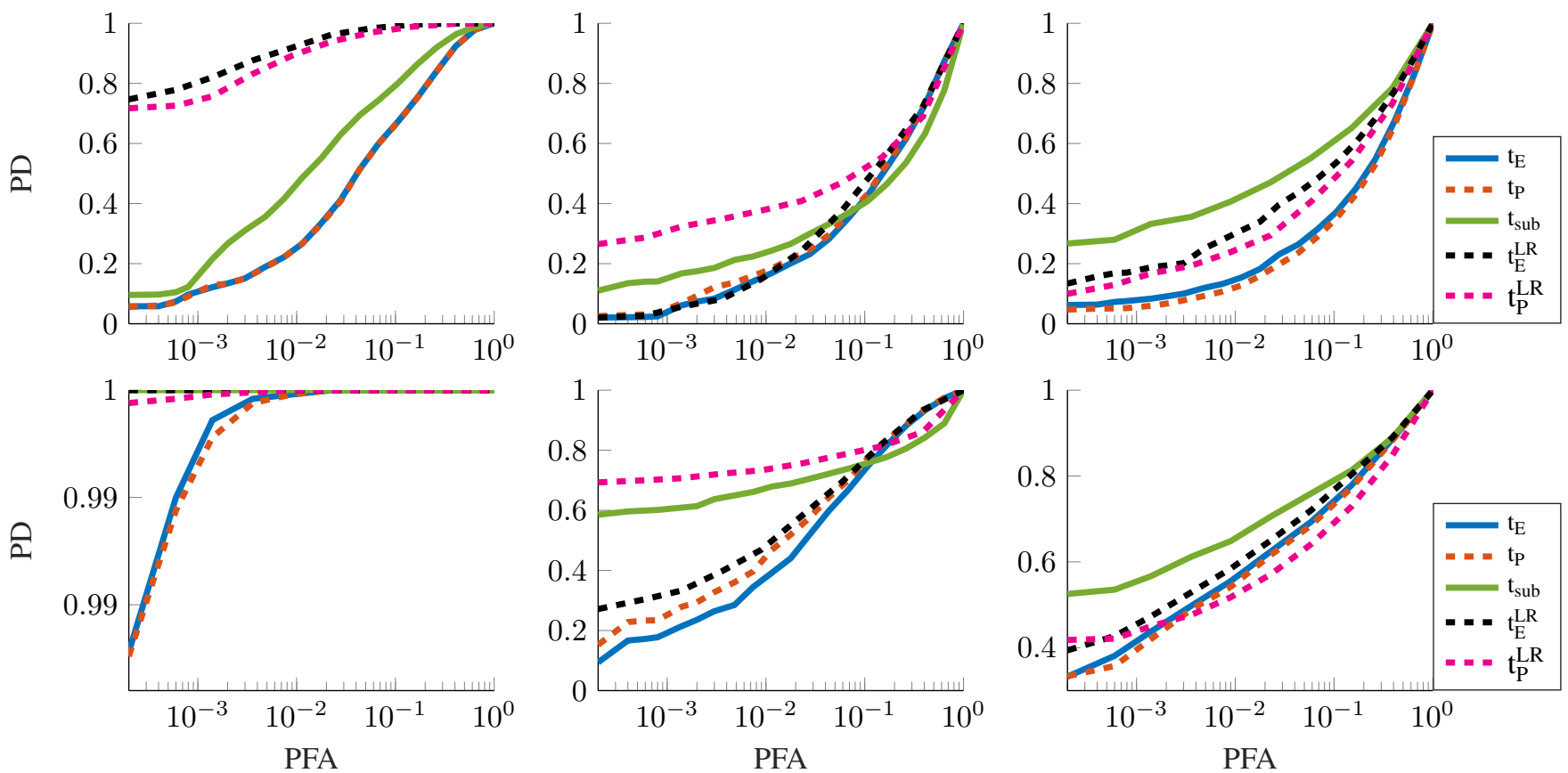

Figure 1. ROC curves for Scenario 1 to 3 (from left to right column) where SNR $=0 \mathrm{~dB}$ (top) and SNR $=5 \mathrm{~dB}$ (bottom).

We proposed to evaluate the corresponding GLRT by making use of the MM algorithm. Finally, numerical simulations illustrated the performance and properties of the proposed detector.

\section{REFERENCES}

[1] P. C. O'Brien, "Robust procedures for testing equality of covariance matrices," Biometrics, pp. 819-827, 1992.

[2] F. T. Walterand et al., "Testing proportionality of covariance matrices," The Annals of Mathematical Statistics, vol. 22, no. 1, pp. 102-106, 1951.

[3] M. S. Srivastava and H. Yanagihara, "Testing the equality of several covariance matrices with fewer observations than the dimension," Journal of Multivariate Analysis, vol. 101, no. 6, pp. 1319-1329, 2010.

[4] D. Ciuonzo, V. Carotenuto, and A. De Maio, "On multiple covariance equality testing with application to SAR change detection," IEEE Transactions on Signal Processing, vol. 65, no. 19, pp. 5078-5091, 2017.

[5] V. Carotenuto, A. De Maio, D. Orlando, and L. Pallotta, "Adaptive radar detection using two sets of training data," IEEE Transactions on Signal Processing, vol. 66, no. 7, pp. 1791-1801, April 2018.

[6] A. A. Nielsen, K. Conradsen, and H. Skriver, "Omnibus test for change detection in a time sequence of polarimetric SAR data," IEEE International Geoscience and Remote Sensing Symposium (IGARSS), 2016.

[7] L. M. Novak, "Change detection for multi-polarization multi-pass SAR," Algorithms for Synthetic Aperture Radar Imagery XII, vol. 5808, no. 1, pp. 234-247, 2005.

[8] V. Carotenuto, A. De Maio, C. Clemente, and J. Soraghan, "Unstructured versus structured glrt for multipolarization SAR change detection," IEEE Geoscience and Remote Sensing Letters, vol. 12, no. 8, pp. 1665-1669, 2015.

[9] A. Taylor, H. Oriot, P. Forster, and F. Daout, "Reducing false alarm rate by testing proportionality of covariance matrices," 2017 International RADAR conference, 2017.

[10] R. Ben Abdallah, A. Mian, A. Breloy, A. Taylor, M. N. El Korso, and D Lautru, "Detection methods based on structured covariance matrices for multivariate sar images processing," IEEE Geoscience and Remote Sensing Letters, 2019.
[11] G. Ginolhac, P. Forster, F. Pascal, and J. P. Ovarlez, "Performance of two low-rank stap filters in a heterogeneous noise," IEEE Transactions on Signal Processing, vol. 61, no. 1, pp. 57-61, 2013.

[12] A. Aubry, A. De Maio, and L. Pallotta, "A geometric approach for structured radar covariance estimation," Radar Conference (RadarConf), 2017 IEEE, pp. 0767-0771, 2017.

[13] Y. Sun, A. Breloy, P. Babu, D. P. Palomar, F. Pascal, and G. Ginolhac, "Low-complexity algorithms for low rank clutter parameters estimation in radar systems," IEEE Transactions on Signal Processing, vol. 64, no. 8, pp. 1986-1998, April 2016.

[14] N. A. Goodman and J. M. Stiles, "On clutter rank observed by arbitrary arrays," IEEE Transactions on Signal Processing, vol. 55, no. 1, pp. 178-186, Jan 2007.

[15] P. Stoica and Y. Selen, "Model-order selection: a review of information criterion rules," IEEE Signal Processing Magazine, vol. 21, no. 4, pp. 36-47, July 2004.

[16] L. Huang and H. C. So, "Source enumeration via mdl criterion based on linear shrinkage estimation of noise subspace covariance matrix," IEEE Transactions on Signal Processing, vol. 61, no. 19, pp. 4806-4821, Oct 2013.

[17] E. Terreaux, J.-P. Ovarlez, and F. Pascal, "Robust model order selection in large dimensional elliptically symmetric noise," arXiv:1710.06735.

[18] Y. Sun, P. Babu, and D. P. Palomar, "Majorization-minimization algorithms in signal processing, communications, and machine learning," IEEE Transactions on Signal Processing, vol. 65, no. 3, pp. 794-816, Feb 2017.

[19] D. R. Hunter and K. Lange, "A tutorial on MM algorithms," The American Statistician, vol. 58, no. 1, pp. 30-37, 2004.

[20] B. Kang, V. Monga, and M. Rangaswamy, "Rank-constrained maximum likelihood estimation of structured covariance matrices," IEEE Transactions on Aerospace and Electronic Systems, vol. 50, no. 1, pp. 501-515, January 2014 